rates were compared to provincial rates using standardized incidence ratios (SIR). Internal comparisons were made using Poisson regression to estimate age and birth year-adjusted incidence rate ratios (RRs) and 95\% confidence intervals (CIs) for NMRDs. Stratified analyses were conducted for types of mining and ore mined.

Results The linked cohort of 30,413 males displayed significantly increased risks for COPD $(\mathrm{SIR}=2.65, \mathrm{CI}=2.58-2.71)$, pulmonary fibrosis $(\mathrm{SIR}=2.02, \mathrm{CI}=1.85-2.20)$, and silicosis $(\mathrm{SIR}=15.72, \quad \mathrm{CI}=14.55-20.65)$. Excesses of silicosis were observed for underground miners $(\mathrm{SIR}=17.41, \mathrm{CI}=14.55-$ 20.65), surface miners $(S I R=19.58, C I=15.20-24.82)$ and mixed-ore miners $(S I R=25.52, C I=20.98-30.76)$. Surface miners had an increased risk of silicosis compared to never surface miners. This risk increased with increasing duration of employment with the highest employment duration $(\geq 10$ years) yielding an RR of 3.49 (CI 1.69-7.21).

Conclusion Results are consistent with previous findings of an excess risk of respiratory disease (NMRD) among Ontario mine workers. This study provides increased understanding of these risks in relation to occupational factors and highlights a potentially overlooked increased risk of silicosis among surface mine workers in Ontario that bears further scrutiny. These results are supported by the unexpectedly high respirable crystalline silica exposures measured in Ontario mines surface operations as part of the related Ontario Mines Exposure Database (OMED). Together with the MMF the OMED exposure data provides future opportunities to test new hypotheses, including the impact of combined exposures among miners.

\section{0-302 A POPULATION PERSPECTIVE FOR THE PREVENTION OF OCCUPATIONAL ASTHMA (EUROPEAN COMMUNITY RESPIRATORY HEALTH SURVEY-ECRHS)}

${ }^{1}$ Manolis Kogevinas, Theodore Lytras, Jan Paul Zock, Paul D Blanc, Simona Villani, Hans Kromhout, Katja Radon, Cecilie Svanes, Kjell Toren, Torben Sigsgaard, Sandra Dorado Arenas, Vivi Schlünssen, Nicole Le Moual, Anne-Elie Carsin, Sheikh Alif, Geza Benke. 'IS GLOBAL, Spain

\subsection{6/OEM-2021-EPI.128}

Introduction About 15\% of adult-onset asthma is attributed to occupational exposures.

Objectives We examined whether prevention policies focusing on high-risk occupations adequately identify occupational asthma risks at a population level. We estimated, in a prospective population-based study, the distribution of asthma risk by occupation, and examined whether asthma risk in prior defined high-risk occupations were distinguished from those of prior defined non-high-risk occupations.

Methods ECRHS is a multicentre cohort study; 9409 participants $(52 \%$ female) from 13 countries were followed for 10 to 20 years. Incident asthma was assessed by repeated questionnaires on asthma symptoms and medication. We examined the incidence of asthma for each occupation relative to all other occupations during follow-up using Generalized Estimating Equation Poisson regression. On the basis of prior evidence, we classified occupations during follow-up into two asthma risk groups (high and non-high risk). All high-risk occupations had documented exposure to at least one highrisk asthmagen (Occupational Asthma-specific Job Exposure Matrix [JEM]). We compared the distributions of the estimated $\log$ Relative Risks (logRR) for each group limiting the main analysis to 90 occupations with more than five incident cases.

Results The median $\log R \mathrm{R}$ for the 14 prior defined high-risk occupations such as bakers, cleaners, and welders was higher $(\log R R=0.37)$ than the non-high risk occupations $(\operatorname{logRR}=0.06) \quad($ Mann-Whitney $\mathrm{p}$-value $=0.02)$. There was considerable overlap in the distributions of the $\operatorname{logRR}$ by risk group (high risk, InteQuartileRange $\operatorname{logRR} 0.14-0.49$; nonhigh risk IQR -0.21 to 0.36). Several non-high risk group occupations had significantly increased logRRs, including architects and receptionists.

Conclusion The significant overlap in risk curves by prior risk indicates that asthma-related exposures are prevalent and occur in multiple occupations. Current programs for occupational asthma prevention focus correctly on occupations at higher risk, but should also consider exposures in occupations not identified, a priori, as high risk.

\section{Return to Work}

\section{0-146 ASSOCIATIONS OF EMPLOYMENT SECTOR AND OCCUPATIONAL EXPOSURES WITH FULL AND PART- TIME SICKNESS ABSENCE: RANDOM AND FIXED EFFECTS ANALYSES ON PANEL DATA}

${ }^{1}$ Elli Hartikainen, Svetlana Solovieva, Eira Viikari-Juntura, Taina Leinonen. ${ }^{1}$ Finnish Institute of Occupational Health, Finland

\subsection{6/OEM-2021-EPI.129}

Introduction The contribution of work-related factors - such as employment sector and occupational exposures - to sickness absence is known to be strong. In addition to the potential causal mechanisms, the associations may also be affected by selection, i.e. individuals with characteristics associated with a high likelihood of sickness absences ending up in particular types of jobs.

Objectives We aimed to investigate the influence of unobserved individual characteristics in explaining the effect of work-related factors on full (fSA) and part-time sickness absence (pSA).

Methods We used register-based panel data for the period 2005-2016 on a 70\% random sample of the Finnish workingage population. The relationships between employment sector and occupational exposures ( $\%$ exposed to physically heavy work and job control score based on job exposure matrices) and the annual onset of fSA and pSA were investigated among men and women. First random effects (RE) models were applied controlling for observed sociodemographic factors and then fixed effects (FE) models, that examine within-individual changes over time and thereby further account for unobserved time-invariant individual characteristics.

Results In the RE analyses, public employment sector, physically heavy work and lower job control each increased the use of fSA and pSA among both genders. When unobserved individual characteristics were controlled for with the FE models, the effects on fSA attenuated. With pSA the effects of employment sector and physical heaviness of work among women even became reverse. The effect of lower job control on pSA remained specially among women.

Conclusions The role of individuals' unobserved characteristics in explaining the effect of work-related factors on sickness absence should not be neglected. The effects of work-related 
factors are likely to be overestimated when using traditional approaches that do not account for unobserved confounding, i.e. selection of individuals with a high likelihood of sickness absence into particular work environments.

\section{0-208 IMMIGRANT WORKERS AND WORK DISABILITY DURATION IN BRITISH COLUMBIA, CANADA}

Sonja Senthanar, 'Mieke Koehoorn, Lillian Tamburic, Stephanie Premji, Ute Bültmann, Chris McLeod. 'University of British Columbia, Canada

\subsection{6/OEM-2021-EPI.130}

Introduction Immigrants are overrepresented in low prestige and precarious employment positions that may expose them disproportionally to work-related injury and illness but also longer work disability durations.

Objective To investigate differences in disability duration among immigrants (categorized as economic, family or refugees/other classification upon arrival to Canada) compared to Canadian-born workers with a work-related injury in British Columbia.

Methods Immigrants and Canadian-born workers were identified from linked workers' compensation claims and immigration records with back strain, connective tissue, concussion and fracture injuries requiring at least one day of work disability between 2009 to 2015 . Quantile regression investigated the relationship between immigration classification and predicted disability days (defined as time on claim within one year of injury) at the 25 th, 50th and 75 th percentile of the distribution.

Results With a few exceptions, immigrants experienced greater predicted disability days compared to Canadian-born workers within the same injury cohort. The largest differences were observed for family and refugee/other immigrant classification workers, and in particular for women within these classifications compared to Canadian-born workers. For example, at the 75 th percentile of the distribution of disability days, we observed a difference of 47.9 days longer for refugee/other women in the concussion cohort and a difference of 41.6 days longer for family classification women in the fracture cohort. Economic immigrants had comparable disability days with Canadian-born workers, especially in the connective tissue and back strain injury cohorts at the 25th and 50th percentiles of the distribution.

Conclusion Immigrant workers' longer disability durations may be a result of more severe injuries, or challenges navigating the workers' compensation system with delays in seeking disability benefits and rehabilitation services. Differences by immigrant classification speaks to vulnerabilities or inequities upon arrival in Canada that persist upon entry to the workforce and warrant further investigation for early mitigation strategies.

\section{0-219 EMPLOYEES RECEIVING INPATIENT TREATMENT FOR COMMON MENTAL DISORDERS IN GERMANY: FACTORS ASSOCIATED WITH TIME TO FIRST AND FULL RETURN TO WORK}

${ }^{1}$ Alexandra Sikora, Gundolf Schneider, Uta Wegewitz, Ute Bültmann. ${ }^{1}$ Federal Institute for Occupational Safety and Health, Germany

10.1136/OEM-2021-EPI.131
Introduction In Germany, return to work (RTW) after inpatient treatment for common mental disorders (CMDs) is a complex process at the intersection of the mental healthcare system and the workplace.

Objectives This study examined 1) the time to first and full RTW and 2) associated factors among employees receiving inpatient treatment for CMDs.

Methods In this prospective cohort study, employees receiving inpatient psychiatric or medical rehabilitation treatment for CMDs were interviewed by phone during their last week before discharge. Follow-up interviews were conducted after 6 , 12 , and 18 months. Health-, personal-, and work-related factors were used from baseline measurement. Parametric survival analysis was conducted to identify factors associated with time to first and full RTW.

Results A total of $\mathrm{N}=269$ participants who stayed at a psychiatric clinic or a medical rehabilitation facility were included. Almost all participants $(\mathrm{n}=252,94 \%)$ from both treatment settings reported a first RTW and a full RTW. The time to first and full RTW was shortest among participants from medical rehabilitation (both median 6 days) and longer among participants from psychiatric treatment (median 17 days to first RTW and 73 days to full RTW). While only health-related and personal factors were associated with time to first RTW, leadership quality and needed individual RTW support were associated with time to full RTW.

Conclusion More attention to work accommodation needs for RTW in clinical practice and coordinated actions towards RTW in collaboration with key RTW stakeholders in the workplace may support a timely RTW.

\section{0-282 ASSOCIATION BETWEEN PATTERNS OF RETURN-TO- WORK TRAJECTORIES AND LONG-TERM DEPRESSIVE SYMPTOMS AMONG BREAST CANCER SURVIVORS}

${ }^{1}$ Mélanie Bertin, Yves Roquelaure, Bertrand Porro, Fabien Gilbert, Clémence Rapicault. 'École des hautes études en santé publique (EHESP), France

\subsection{6/OEM-2021-EPI.132}

Introduction Depressive symptoms and fatigue are well known factors affecting return-to-work (RTW) after breast cancer (BC). However, the RTW process may in turn have a positive impact on long-term health and psychological well-being among breast cancer survivors (BCS).

Objective Our aim was to identify RTW trajectories within the 5 -years following BC using a multi-phase and diachronic process and to assess their associations with depressive symptoms measured at least five year after their $\mathrm{BC}$ diagnostic.

Methods We used data from the French Constances cohort that included more than 200,000 participants from 2012 to 2020. Our study relied on a sub-sample of women aged up to 55 years at the time of their diagnostic, who were working at the time of their diagnostic and who fully completed their occupational calendar up to five years after their diagnostic $(n=939)$. Sequence analysis was used to identify RTW trajectories among BCS from their diagnosis up to 5 years later. Depressive symptoms were assessed using the 20 -items CES-D scale. Adjusted logistic regression analyses were performed to assess the association between RTW trajectories and depressive symptoms.

Results In our sample, $12.8 \%$ of BCS suffered from depressive symptoms at their inclusion in the cohort. Four types of 